\title{
Statistical analysis of a self-seeded x-ray free-electron laser in the presence of the microbunching instability
}

\author{
Zhen Zhang®, ${ }^{*}$ Gabriel Marcus, ${ }^{\dagger}$ Erik Hemsing $\odot,{ }^{\ddagger}$ William M. Fawley, \\ Zhirong Huang, and Alberto Lutman \\ SLAC National Accelerator Laboratory, Menlo Park, California 94025, USA
}

(Received 15 October 2019; published 21 January 2020)

\begin{abstract}
Self-seeding is one of the most promising methods to improve the longitudinal coherence and spectral purity of free-electron lasers (FELs). Measurements of the multishot-averaged, soft x-ray self-seeding spectrum at the Linac Coherent Light Source (LCLS) FEL often have a pedestal-like distribution around the seeded wavelength. In this paper, a theoretical model based on the mechanism of spectral sideband generation is developed to explain the statistical behavior of the seed and pedestal. The model is in good agreement with statistical analysis of numerical FEL simulations and experimental measurements that show that pedestal fluctuations reflect the level of long-wavelength microbunching structures in the beam, and are driven by fluctuations of the seed.
\end{abstract}

DOI: 10.1103/PhysRevAccelBeams.23.010704

\section{INTRODUCTION}

The self-seeding scheme [1-7] has been demonstrated to increase the longitudinal coherence of x-ray FELs without requiring the use of an external laser seed. In this scheme, the self-amplified spontaneous emission (SASE) from upstream undulator sections is filtered to a narrow bandwidth and used to seed downstream undulator sections. At soft x-ray (SXR) wavelengths, a conventional grating monochromator serves as the filter. A magnetic chicane between the two undulator stages acts to wash out any residual SASE bunching structure while also diverting the electron beam around the x-ray optics, and providing a delay to establish longitudinal overlap with the filtered seed radiation. The downstream FEL sections are sufficiently long for the new narrow-bandwidth FEL radiation to reach full saturation.

Recent SXR self-seeding measurements at the LCLS $[5,8]$ show that the narrow spectral line of the amplified seed can also be surrounded by a broad spectral pedestal that fills the FEL bandwidth, especially when the FEL power approaches saturation levels. The wavelengthintegrated pedestal strength can approach $20 \%$ or greater of the total integrated pulse energy, a portion of which can be attributed to a SASE background. However, there are a

\footnotetext{
*zzhang@slac.stanford.edu

gmarcus@slac.stanford.edu

ehemsing@slac.stanford.edu
}

Published by the American Physical Society under the terms of the Creative Commons Attribution 4.0 International license. Further distribution of this work must maintain attribution to the author(s) and the published article's title, journal citation, and DOI. number of strong indications that the pedestal also includes a sideband component driven by frequency mixing of the amplified seed radiation with the microbunchinginstability-induced ( $\mu$ BI-induced) modulations in the electron beam $[9,10]$. These indications include [8]: (1) a pedestal strength that decreases with increasing laser heater (LH) strength, (2) a positive correlation between the strengths of pedestal wavelength components which are symmetrically offset from the central seed wavelength, and (3) the pedestal strength approaches that corresponding to normal SASE emission when the seed strength is strongly decreased by detuning the electron beam energy from resonance.

The growth rate of the $\mu \mathrm{BI}$-induced pedestal components along the undulator has been studied previously with onedimensional FEL theory, where it was shown that the relative growth rate scales quadratically with the undulator length [11]. This relation has been confirmed by the threedimensional simulation and a new method was proposed to suppress the pedestal [12]. However, the connection between the statistical energy fluctuations of the seed and that of the pedestal has been less clear. One issue in particular is whether shot-to-shot fluctuations in the pedestal (e.g., arising from fluctuations in the $\mu \mathrm{BI}$ power spectrum) can drive fluctuations in the amplified seed. Here we find the opposite is true: fluctuations in the seed drive fluctuations in the spectral pedestal, but microbunching fluctuations have little impact on the seed fluctuations.

In this paper, we study the statistical properties of the self-seeding XFEL output generated by an electron beam with $\mu \mathrm{BI}$-induced structure. Analysis of the experimentally measured spectra at the LCLS suggests a correlation between the statistical properties of the spectral pedestal 
and the level of $\mu \mathrm{BI}$ in the electron beam. To explain this correlation, we develop theoretical models and then perform numerical simulations. In Sec. II, we first present the theoretical models with some definitions and notation that are used for the statistical analysis. Numerical simulations are shown in Sec. III with different conditions of $\mu$ BI to verify the theoretical models. Lastly in Sec. IV, we present the results of the statistical analysis for the recent selfseeding spectra obtained at the LCLS [8].

\section{THEORETICAL ANALYSIS}

The statistical properties of SASE FELs have been rigorously studied in the past (e.g., see [13-17] and references therein) where it was found that the radiation was characteristic of a single polarized chaotic source. The SASE FEL, therefore, can be described rather well within the framework of statistical optics [18]. In this paper, however, we study the statistical properties of self-seeded radiation in the presence of strong $\mu \mathrm{BI}$-induced electron beam modulations. The radiation produced and amplified downstream of the self-seeding monochromator is therefore potentially composed of three distinct sources each with potentially different statistical properties. First, the monochromatized SASE radiation from the upstream undulators, the seed, may not be transform limited if the Fourier-limited pulse length of the monochromator bandwidth is shorter than the length of the electron beam. That is, multiple SASE frequency spikes can be transmitted through the monochromator, which modifies the statistics compared to a single frequency spike. Second, the seed radiation frequency-mixes with the modulations on the electron beam to drive the amplification of spectral components outside of the primary seeded region. These spectral components constitute on average what is often referred to as the spectral pedestal (see Sec. II B below). Third, SASE radiation is produced in the downstream postmonochromator undulators that is uncorrelated with the monochromatized SASE radiation from the upstream undulators [19]. This occurs because the magnetic chicane that bridges the upstream and downstream undulator sections resets the shot noise in the electron beam that is assumed to be uncorrelated with the initial shot noise. These three sources of radiation together influence the spectral fluctuations seen by a post-undulator spectrometer.

At a fixed undulator position before saturation, consider the integrated energy within a specified bandwidth in a single pulse,

$$
W \propto \int_{\omega_{0}-\Delta \omega / 2}^{\omega_{0}+\Delta \omega / 2} \tilde{I}(\omega) d \omega
$$

where $\omega_{0}$ and $\Delta \omega$ are respectively the center frequency and bandwidth of interest and $\tilde{I}(\omega)$ is the instantaneous spectral power density. The fluctuation $(\sigma)$ of the energy within this bandwidth defines the effective number of modes $M$,

$$
\sigma^{2}=\frac{\left\langle(W-\langle W\rangle)^{2}\right\rangle}{\langle W\rangle^{2}} \equiv \frac{1}{M} .
$$

We note that for a single polarized chaotic source, this definition relates directly to the fluctuations described by a gamma probability density distribution

$$
\begin{aligned}
p_{W}(W)= & \frac{M_{\Gamma}^{M_{\Gamma}}}{\Gamma\left(M_{\Gamma}\right)}\left(\frac{W}{\langle W\rangle}\right)^{M_{\Gamma}-1} \\
& \times \frac{1}{\langle W\rangle} \exp \left(-M_{\Gamma} \frac{W}{\langle W\rangle}\right),
\end{aligned}
$$

where $M_{\Gamma}$ is the number of modes according to the gamma distribution. In this paper, however, we study the statistical properties of the self-seeding FEL output with $\mu \mathrm{BI}$, which models the sum and product of multiple chaotic sources. In this case, it is not clear a priori that the resultant probability distribution is well modeled by a gamma function and its associated mode number. Consequently, for the remainder of this paper we refer to the effective number of modes $M$ as determined from the normalized fluctuation $\sigma$ via Eq. (2).

\section{A. Mode analysis at the seed frequency}

We first consider the statistical behavior of the seed spectrum in the presence of a small SASE background pedestal, but in the absence of $\mu \mathrm{BI}$. Let the center of the integration bandwidth $\omega_{0}$ in Eq. (1) be set to the seed frequency. We can assume that the total output energy $(W)$ includes the seed signal $\left(W_{1}\right)$ and the SASE radiation $\left(W_{0}\right)$ only from the second stage. The ensemble-averaged total energy is

$$
\langle W\rangle=\left\langle W_{1}\right\rangle+\left\langle W_{0}\right\rangle
$$

The spectrum of the seed is set by the effective transmission bandwidth of the monochromator $\sigma_{m}$ used in the experiments [13], which we presume to have a Gaussian profile. The SASE radiation from the second stage is characterized by the corresponding FEL bandwidth, $\sigma_{A} \gg \sigma_{m}$. According to the definition of fluctuations in Eq. (2), the effective number of total modes $M$ can be derived as a function of the integration bandwidth $\Delta \omega$ with the assumption of independent sources, i.e., $\left\langle\left(W_{1}-\left\langle W_{1}\right\rangle\right)\left(W_{0}-\left\langle W_{0}\right\rangle\right)\right\rangle=0$,

$$
M=\frac{\left(\left\langle W_{1}\right\rangle+\left\langle W_{0}\right\rangle\right)^{2}}{\left\langle W_{1}\right\rangle^{2} / M_{1}+\left\langle W_{0}\right\rangle^{2} / M_{0}} .
$$

Here $M_{0}$ and $M_{1}$ are the effective numbers of modes within the bandwidth $\Delta \omega$ of the SASE and seed radiation at the second stage, respectively. For $\Delta \omega \rightarrow \infty$ we can assume $M_{0} \gg M_{1}$.

By allowing $\Delta \omega$ to vary, we can study and compare the statistical properties of the two sources that have different 
bandwidths and intensities. For example, from the energy fluctuations within a narrow window around the seed spike, one can determine the number of coherent seed modes $M_{1}$. For a rectangular electron beam temporal profile, the smallest spectral interval of coherence is inversely proportional to the electron bunch duration $T_{b}$,

$$
\Omega=\frac{2 \pi}{T_{b}} .
$$

When the integration window is smaller than the spectral interval of coherence, $\Delta \omega<\Omega / 2 \pi$, the individual mode numbers approach unity,

$$
M_{0}=1, \quad M_{1}=1
$$

In this regime, the total number of modes as expressed by Eq. (5) is

$$
M=\frac{\left(\left\langle W_{1}\right\rangle+\left\langle W_{0}\right\rangle\right)^{2}}{\left\langle W_{1}\right\rangle^{2}+\left\langle W_{0}\right\rangle^{2}} .
$$

Note that $1 \leq M \leq 2$, and it is never unity unless either source vanishes. Consequently, the presence of SASE will always make it appear that there is more than one mode in the seed.

If the frequency integration window is larger than that corresponding to spectral coherence width but smaller than the seed bandwidth, $\Omega / 2 \pi<\Delta \omega<\sigma_{m}$, both the mode numbers are identical and grow linearly with $\Delta \omega$,

$$
M_{0}=M_{1}=\frac{\Delta \omega}{\Omega} .
$$

Normally, the energy of the seed is much larger than that of SASE in this range, so the effective number of total modes in Eq. (5) can be simplified as

$$
M \approx M_{1}=\frac{\Delta \omega}{\Omega} .
$$

When the window is larger than the seed bandwidth, but smaller than the full SASE bandwidth, $\Omega / 2 \pi<\sigma_{m}<$ $\Delta \omega<\sigma_{A}$, the number of modes in the amplified seed is then defined by the monochromator bandwidth, while the number of modes contributed by SASE depends on the window width,

$$
M_{0}=\frac{\Delta \omega}{\Omega}, \quad M_{1}=2 \sqrt{\pi} \frac{\sigma_{m}}{\Omega} .
$$

Within this window the contribution of the SASE power in the pedestal can impact the total number of modes, which becomes approximately,

$$
M \approx \frac{\left(\left\langle W_{1}\right\rangle+\left\langle W_{0}\right\rangle\right)^{2}}{\left\langle W_{1}\right\rangle^{2}} \frac{2 \sqrt{\pi} \sigma_{m}}{\Omega} .
$$

Thus as $\Delta \omega$ increases, the effective number of modes gradually increases due to the inclusion of the SASE outside of the monochromator bandwidth. Finally, when the window is much larger than the FEL gain bandwidth, $\Omega / 2 \pi<\sigma_{m} \ll \sigma_{A} \ll \Delta \omega$, the individual modes reach their asymptotic values

$$
M_{0}=2 \sqrt{\pi} \frac{\sigma_{A}}{\Omega}, \quad M_{1}=2 \sqrt{\pi} \frac{\sigma_{m}}{\Omega} .
$$

\section{B. Analysis for the pedestal}

Let us now consider the effective number of modes in the spectral pedestal when the contribution from $\mu \mathrm{BI}$-induced sidebands is also included. Because the $\mu \mathrm{BI}$ structures in the beam are typically down to visible wavelengths, $\mu \mathrm{BI}-$ induced sideband emission is usually outside the SXR monochromator bandwidth. Thus, to ascertain the statistical properties of the pedestal with $\mu \mathrm{BI}$ included, we take the center of integration in Eq. (1) to be shifted away from the seed frequency such that we may neglect any amplified seed contributions and consider only contributions from SASE and sidebands in this displaced spectral bandwidth. The average pedestal energy within this window is

$$
\left\langle W_{\text {ped }}\right\rangle=\left\langle W_{0}\right\rangle+\left\langle W_{s}\right\rangle .
$$

The theoretical model presented in Ref. [11] indicates that within a certain frequency range detuned from the seed, the energy in the sidebands $W_{s}$ is proportional to the seed energy $W_{1}$ and the square of the $\mu \mathrm{BI}$ modulation amplitude $|A|^{2}$ at the corresponding wavelength:

$$
W_{s} \propto W_{1}|A|^{2} .
$$

According to the definition of fluctuations in Eq. (2), the effective number of modes in the sideband $M_{s}$ is related to the number of modes in the seed $M_{1}$ and the "modes" in the $\mu \mathrm{BI}$-induced modulations $M_{\mu \mathrm{BI}}$,

$$
\frac{1}{M_{s}}=\frac{1}{M_{1}}+\frac{1}{M_{\mu \mathrm{BI}}}+\frac{1}{M_{1} M_{\mu \mathrm{BI}}} .
$$

This relation is shown explicitly in the Appendix. Here $M_{\mu \mathrm{BI}}$ is obtained directly from the fluctuations in the microbunching spectrum at the wavelength that produces the spectral sideband. We see that the effective number of modes of the sideband $M_{s}$ is always smaller than either $M_{1}$ or $M_{\mu \mathrm{BI}}$, and it will generally be determined by the smaller of the two. With this result for $M_{s}$, the effective number of modes in the spectral pedestal $M_{\text {ped }}$ can be written as 


$$
M_{\text {ped }}=\frac{(1+\eta)^{2} M_{0} M_{s}}{M_{s}+\eta^{2} M_{0}},
$$

where $\eta$ is defined as the ratio between the sideband and SASE contributions to the pedestal $\eta=\left\langle W_{s}\right\rangle /\left\langle W_{0}\right\rangle$. When the electron beam has large $\mu$ BI-induced modulations and the FEL's spectral pedestal is dominated by the sidebands rather than SASE ( $\eta \gg 1$ ), the effective number of pedestal modes is naturally approximately the same as the number of sideband modes

$$
M_{\text {ped }} \approx M_{s} .
$$

On the other hand, when $\eta \ll 1$, the effective number of pedestal modes approaches the SASE value,

$$
M_{\text {ped }} \approx M_{0} .
$$

Between the two limits, $M_{\text {ped }}$ varies from $M_{s}$ to $M_{0}$. Thus, from Eq. (17), the statistics of the spectral pedestal are governed by both the SASE statistics and the sideband statistics, as well as by the relative level of microbunching in the electron beam.

\section{Effects from fluctuations of electron beam parameters}

In addition to the fluctuations from the properties of the FEL seeding process, there are also many other sources that will affect the fluctuations of the self-seeding output, including shot-to-shot variations of the electron beam parameters. Here we study the shot-to-shot peak current variations as an example. Considering the case of pure SASE after many exponential gain lengths of growth, the FEL intensity coefficient scales as

$$
A_{0} \propto e^{\sqrt{3} \hat{z}}
$$

where $\hat{z}=2 \rho k_{u} z$ is the scaled distance along the undulator, $2 \pi / k_{u}$ is the undulator period and $\rho$ is the FEL Pierce parameter [20]. The parameter $\rho$ scales with $I_{0}^{1 / 3}$, and thus the SASE intensity in the exponential gain regime will fluctuate as

$$
\frac{\Delta A_{0}}{A_{0}}=\frac{\hat{z}}{\sqrt{3}} \frac{\Delta I_{0}}{I_{0}} .
$$

The resulting modified number of effective SASE modes is then

$$
M_{0} \rightarrow \frac{M_{0}}{1+\frac{\left\langle\left(\Delta A_{0}\right)^{2}\right\rangle}{A_{0}^{2}}\left(M_{0}+1\right)} .
$$

We see that the current fluctuations reduce the effective mode number from that expected in the absence of any such fluctuations. Therefore, as previously concluded by Yurkov [21], simultaneous measurements of such critical beam parameters influencing the FEL process must be performed for accurate comparison with the theoretical model for SASE. Analysis shows that an identical expression as Eq. (22) can be written for the number of seed modes. However, because $M_{0} \gg M_{1}$, the SASE shot-to-shot fluctuation level is significantly more sensitive to beam current fluctuations than that of the amplified seed.

\section{SIMULATION RESULTS}

Time-dependent 1D FEL simulations were performed for different $\mu \mathrm{BI}$ and initial seed signals to study the number of modes in the self-seeding XFEL output and compare with the analytic description. Here we adopt parameters (see Table I) similar to a recent soft $\mathrm{x}$-ray $(1 \mathrm{keV})$ self-seeding study conducted at LCLS [8].

In the simulations, the electron beam (with or without $\mu \mathrm{BI}$-induced density and energy modulations) first generates strong SASE with 7.4 MW average power in the first stage of undulator. It then passes through a magnetic chicane that disperses the electron beam and resets the shot noise, and the SASE radiation is filtered by a secondorder super-Gaussian monochromator with FWHM bandwidth $200 \mathrm{meV}$. The simulated spectra before and after the monochromator are shown in Fig. 1. In the second stage of undulator, the filtered radiation interacts with the electron beam and is amplified through the FEL process. The monochromator efficiency can be set to be zero to perform a SASE simulation in the second stage undulators. Otherwise, the nominal bandwidth and $4 \%$ efficiency are based on the current monochromator parameters at LCLS $[5,8]$.

To simulate the electron beam with $\mu \mathrm{BI}$, we modulate the electron beam current and energy according to measured $\mu \mathrm{BI}$ spectra at the LCLS with $\sim 2 \mu \mathrm{m}$ peak wavelength and $\sim 0.5 \mu \mathrm{m}$ cutoff wavelength [22]. The modulation

TABLE I. Simulation parameters in 1-D FEL code.

\begin{tabular}{lcc}
\hline \hline Parameter & Value & Unit \\
\hline Beam energy $E_{e}$ & 4.78 & $\mathrm{GeV}$ \\
Slice energy spread $\sigma_{e}$ & 1.2 & $\mathrm{MeV}$ \\
Normalized emittance $\varepsilon_{N}$ & 0.9 & $\mu \mathrm{m}$ \\
Beam current $I$ & 1.4 & $\mathrm{kA}$ \\
Bunch length (flat-top) $L_{t}$ & 60 & $\mathrm{fs}$ \\
Resonance wavelength $\lambda_{r}$ & 1.22 & $\mathrm{~nm}$ \\
Photon energy $E_{\mathrm{ph}}$ & 1.02 & $\mathrm{keV}$ \\
Undulator period $\lambda_{u}$ & 3.0 & $\mathrm{~cm}$ \\
Undulator peak strength $K$ & 3.5 & \\
FEL parameter $\rho$ & $6.95 \times 10^{-4}$ & \\
Gain length $L_{G}$ & 1.98 & $\mathrm{~m}$ \\
Monochromator FWHM bandwidth & 200 & $\mathrm{meV}$ \\
Monochromator efficiency & 0.04 & \\
Chicane R56 & 300 & $\mu \mathrm{m}$ \\
\hline \hline
\end{tabular}




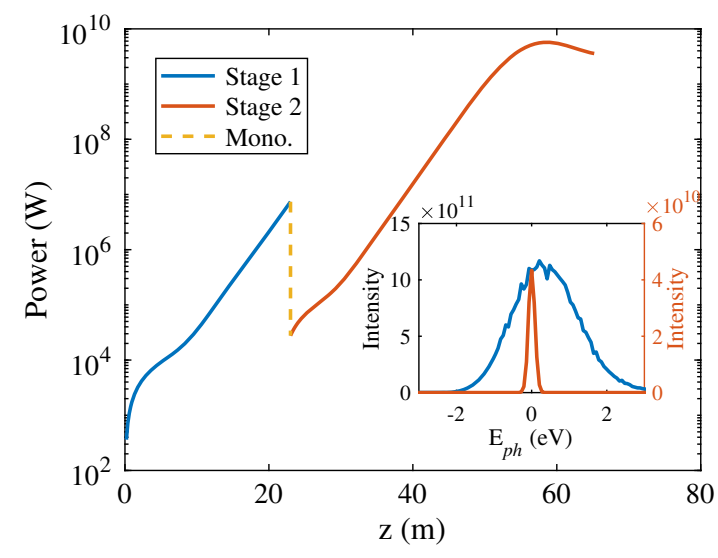

FIG. 1. Average FEL power along the undulator in the simulations of the self-seeding mode. A monochromator is applied between the two stages. The inserted figure shows the spectra before (blue) and after (red) the monochromator. The power and spectra are both averaged over 500 shots.

amplitude is scaled corresponding to different beam conditions. For simplicity, we first assume a hypothetical $\mu \mathrm{BI}$ spectrum with central wavelength $2 \mu \mathrm{m}$ and peak amplitude $b_{\mu \mathrm{BI}}$, which is shown in Fig. 2. For each wavelength in the spectrum, we add a sinusoidal density modulation with random phase to the beam current profile. The modulation amplitude is set according to the theoretical $\mu \mathrm{BI}$ gain spectrum $[9,10,22]$. Figure 2 presents a typical current profile. The corresponding single-shot bunching spectrum and the average bunching spectrum over 500 shots are also given for comparison. The spiky single-shot bunching spectrum is similar with the measured data in Ref. [22]. The energy modulation can also be added based on the density bunching. Namely, assuming the electron beam travels through a long drift before entering the undulator (150 $\mathrm{m}$ in the simulation), the longitudinal space charge

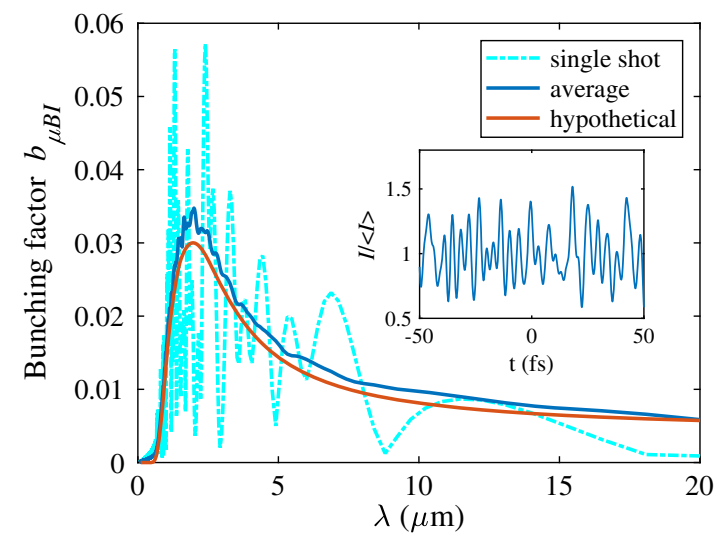

FIG. 2. The hypothetical, one typical single-shot and the average (500 shots) bunching factor spectra of the $\mu \mathrm{BI}$. The peak amplitude of the hypothetical spectrum $b_{\mu \mathrm{BI}}=0.03$. The inserted figure shows the current profile that corresponds to the single-shot bunching spectrum. force (and its associated energy modulation) can be calculated from the derivative of the current profile [23].

Using the methods described above, we performed numerical simulations with different $\mu \mathrm{BI}$ conditions and beam shot noise. The resultant FEL spectra, averaged over 500 shots, are shown in Fig. 3. The spectra are extracted at identical undulator locations ( $z=48 \mathrm{~m}$ in Fig. 1), where the FEL is clearly in the linear regime before saturation. It can be seen that the $\mu \mathrm{BI}$-induced sidebands increase the pedestal level significantly and contaminate the spectral purity. The inserted figure in Fig. 3 shows the fractional spectral energy as a function of integration bandwidth centered at the seed photon energy. The inclusion of $\mu \mathrm{BI}$ decreases the energy fraction from $70 \%$ to $56 \%$ over the bandwidth from $-0.2 \mathrm{eV}$ to $0.2 \mathrm{eV}$. Note that the highenergy pedestal in the self-seeding spectrum is smaller than the low-energy pedestal. This is because the phase difference between the energy and density modulations in the $\mu \mathrm{BI}$ model results in the additional growth at low-energy sidebands and partial suppression at high-energy sidebands [11].

In our theoretical analysis, the number of modes in the spectra without $\mu \mathrm{BI}$ can be calculated by three methods. The first is based on the spectral bandwidths $\sigma_{m}$ and $\sigma_{A}$ given in Eqs. (11) and (13). The root-mean-square (rms) bandwidths obtained by Gaussian fitting the self-seeding and SASE spectra in Fig. 3 are $\sigma_{m}=88 \mathrm{meV}$ and $\sigma_{A}=830 \mathrm{meV}$, respectively. The calculated number of modes $M_{1}$ and $M_{0}$ are shown in the top row of Table II. To calculate the total number of modes from Eq. (5), we integrate the spectra to obtain the average energy from each source. The integrated spectral energy ratio of SASE and seed $\left(\left\langle W_{0}\right\rangle /\left\langle W_{1}\right\rangle\right)$ is 0.12 within the full seed bandwidth $\left( \pm 3 \sigma_{m}\right)$ and 0.62 within the full SASE bandwidth $\left( \pm 3 \sigma_{A}\right)$.

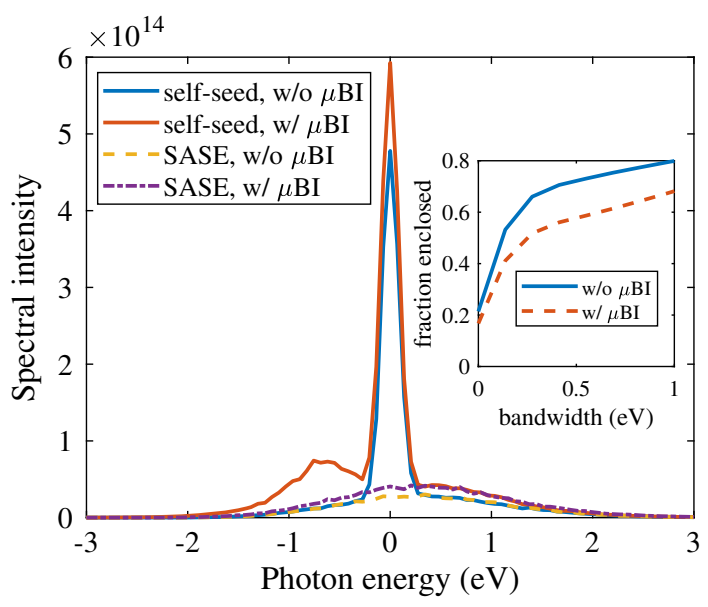

FIG. 3. The average spectra over 500 shots of self-seeding and SASE with or without $\mu \mathrm{BI}$ at the same undulator position $z=48 \mathrm{~m}$. The inserted figure shows the fractional spectral energy as a function of integration bandwidth. The peak amplitude of the hypothetical bunching spectrum $b_{\mu \mathrm{BI}}=0.03$. 
TABLE II. The number of modes of the SASE $\left(M_{0}\right)$, seed $\left(M_{1}\right)$ and sum signal $(M)$ at full seed $\left( \pm 3 \sigma_{m}\right)$ and full SASE $\left( \pm 3 \sigma_{A}\right)$ bandwidth.

\begin{tabular}{|c|c|c|c|c|c|c|}
\hline \multirow{3}{*}{$\begin{array}{l}\Delta \omega \\
<W_{0}>/<W_{1}>\end{array}$} & \multicolumn{3}{|c|}{ $\pm 3 \sigma_{m}$} & \multicolumn{3}{|c|}{ $\pm 3 \sigma_{A}$} \\
\hline & \multicolumn{3}{|c|}{0.12} & \multicolumn{3}{|c|}{0.62} \\
\hline & $M_{0}$ & $M_{1}$ & $M$ & $M_{0}$ & $M_{1}$ & $M$ \\
\hline Bandwidth & 7.7 & 4.5 & 5.7 & 42.8 & 4.5 & 11.3 \\
\hline Fluctuation & 7.5 & 4.4 & 5.5 & 43.0 & 4.3 & 10.8 \\
\hline$\Gamma$-fitting & 7.6 & 4.3 & 5.3 & 40.4 & 4.1 & 10.3 \\
\hline
\end{tabular}

The calculated $M$ values are also shown in Table II, and can be compared with the values calculated with the second method. The second method uses the fluctuations of the spectra within the given bandwidth and Eq. (2) to calculate the effective number of modes. In this case, the effective number of modes in the SASE and sum signal are calculated directly from the simulation data while the values of the pure seed can be derived from Eq. (5). Lastly, the number of modes can also be estimated by fitting the $\Gamma$-distribution in Eq. (3) to the histograms of the simulation data to get the number of modes of SASE and total signal. The mode numbers of the pure seed can be also derived by Eq. (5). It can be observed from Table II that the three methods give similar values for the number of modes without $\mu \mathrm{BI}$ in the beam. Figure 4 shows the normalized (to average) integrated spectral intensity over the full SASE bandwidth histograms (blue bars) of 500-shot self-seeding and SASE output without $\mu$ BI. Empirical gamma function fits (red curves) using Eq. (3) are also presented for comparison.

The effective number of modes will be different when there is $\mu \mathrm{BI}$ in the beam, as indicated by Eq. (17). Figure 5 shows the effective number of modes in the self-seeding and SASE spectra with and without $\mu \mathrm{BI}$ as a function of
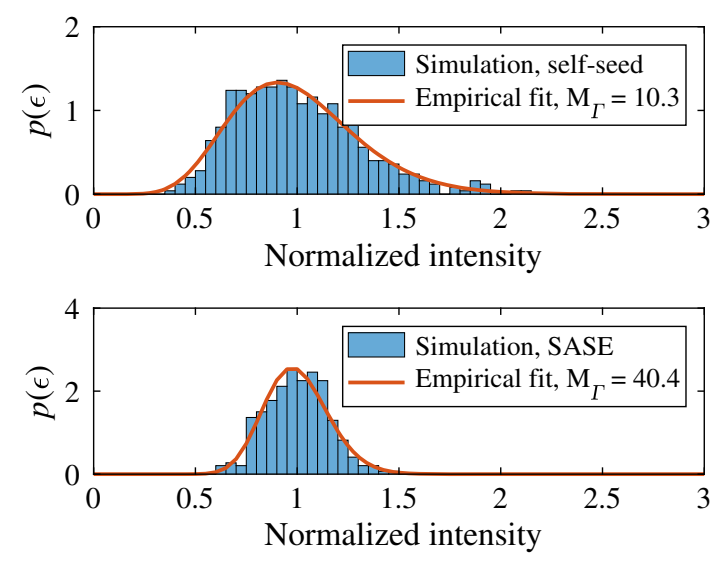

FIG. 4. Histograms of the normalized integrated spectral intensity within full SASE bandwidth (blue bars) and empirically derived probability density function (red curves) using Eq. (3) for self-seeding (top) and SASE (bottom) at $z=48 \mathrm{~m}$.
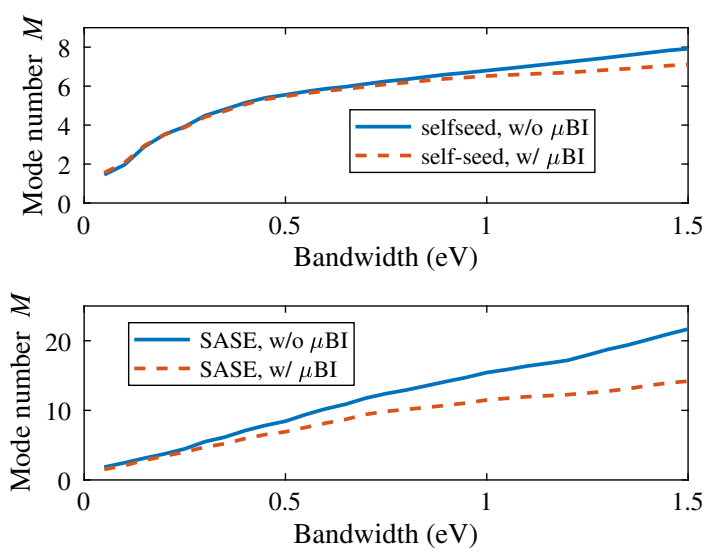

FIG. 5. The effective number of modes $M$ as a function of the integration bandwidth for self-seeding and SASE with or without $\mu \mathrm{BI}$. The center of the integration bandwidth is the photon energy of the seed. The peak amplitude of the hypothetical bunching spectrum $b_{\mu \mathrm{BI}}=0.03$ for the cases with $\mu \mathrm{BI}$.

integration bandwidth $\Delta \omega$. The center of the integration bandwidth $\omega_{0}$ is fixed at the photon energy of the seed signal. In the self-seeding scheme, the bandwidth where the slope starts to change roughly equals the full width of the seed spectrum. We see that $\mu \mathrm{BI}$ has little effect on the mode number of the seed signal, even though there are significant current modulations in the beam (see Fig. 2). For pure SASE, however, the $\mu \mathrm{BI}$ has a stronger effect; it reduces the number of the modes (i.e., increases the fluctuations), especially at a large bandwidth. When viewing $\mu \mathrm{BI}$ as current fluctuations along the beam, this is consistent with the expectation of Eq. (22) in which SASE is predicted to be more sensitive to current fluctuations because $M_{0} \gg M_{1}$.

The statistical analysis can be performed for spectral regions that are detuned from the main seed spike, i.e., the spectral pedestals. As shown in Fig. 3, the low-energy spectral pedestal maximizes around $-0.7 \mathrm{eV}$ offset from the seed. For this spectral region, the effective number of modes are shown in Fig. 6 for different $\mu$ BI conditions.

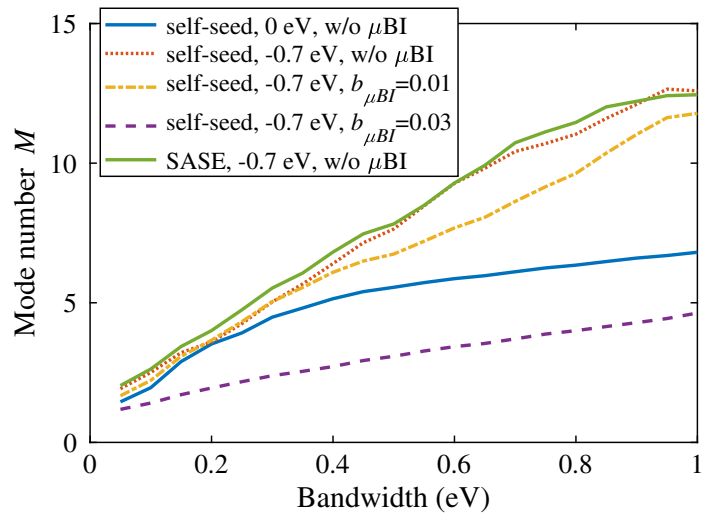

FIG. 6. The effective number of modes $M$ as a function of the integration bandwidth for different conditions listed in the legends. 


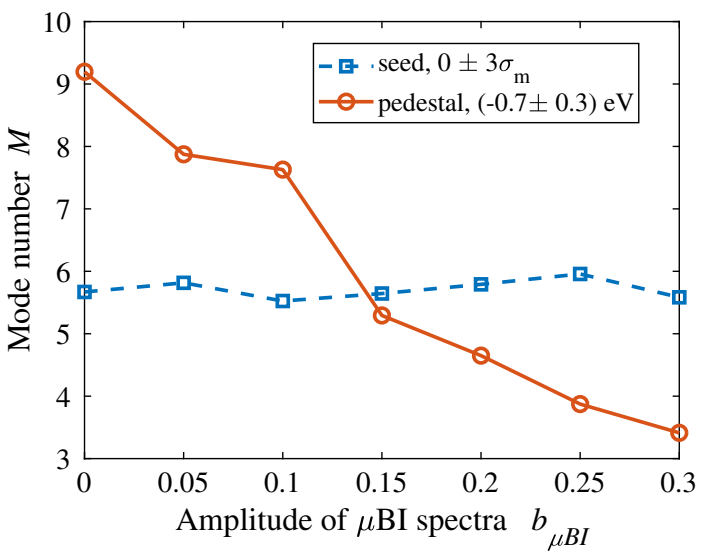

FIG. 7. The effective number of modes $M$ of seed and pedestal as a function of the amplitude of the hypothetical $\mu \mathrm{BI}$ spectra.

When there is no $\mu \mathrm{BI}$, the pedestal mode number is very close to that of SASE. As the $\mu \mathrm{BI}$ increases, the number of modes in the pedestal decreases from the SASE value until the pedestal is dominated by the sidebands. At this point, the number of modes in the pedestal is smaller than the number of modes in the seed, i.e., $\mu \mathrm{BI}$ increases the fluctuations in the pedestal. It does not, however, increase the fluctuations in the seed. This is shown in Fig. 7, where the number of pedestal modes are plotted versus the amplitude of the modeled $\mu \mathrm{BI}$ spectra. There is a clear decrease in the pedestal mode number as the $\mu \mathrm{BI}$ amplitude increases. The statistics of the seed, however, are unaffected by the $\mu \mathrm{BI}$ strength in the electron beam. The number of modes in the seed stays approximately constant as the $\mu \mathrm{BI}$ amplitude grows.

The simulation results above demonstrate the behavior of the effective number of modes in the pedestal under different $\mu \mathrm{BI}$ conditions. In order to compare with the theoretical model for the sideband statistics quantitatively, [Eq. (16), for example] we performed two sets of ideal simulations using a fresh beam with controlled $\mu \mathrm{BI}$, but without shot noise in the amplified seed stage. Thus, there is no SASE background. This situation corresponds to the $\eta \gg 1$ limit described by Eq. (18), where the number of pedestal modes is predicted to match the number of sideband modes, $M_{\text {ped }}=M_{s}$. The beam and undulator parameters are the same as in Table I, and the spectra of the radiation are output at the same undulator length. The first set of simulations employed normal typical self-seeding. In the absence of $\mu \mathrm{BI}$, the fluctuations of the spectra all come from the seed, so the effective number of modes is $M_{1}$. This is shown as the red solid line in Fig. 8. For comparison, in the second set of simulations we adopt a constant and uniform seed whose power equals the average power of the seed signals in the first set of simulations. In this case, the fluctuations of the spectra all come from the $\mu \mathrm{BI}$, so from Eq. (16), the modes in the sideband equal the modes in microbunching $M_{s}=M_{\mu \mathrm{BI}}$. Accordingly, because $\mu \mathrm{BI}$

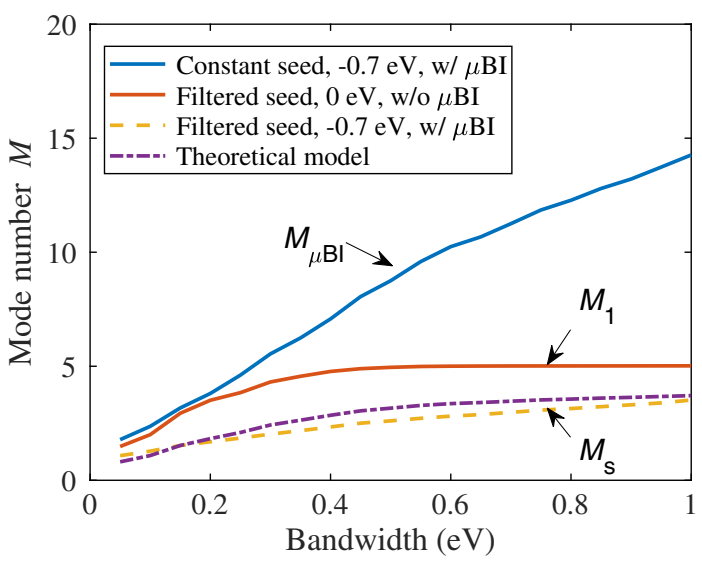

FIG. 8. The effective number of modes $M$ as a function of the integration bandwidth under the conditions: constant seed with $\mu \mathrm{BI}$, fluctuating seed without $\mu \mathrm{BI}$, fluctuating seed with $\mu \mathrm{BI}$. The dotted line is the effective number of modes of the spectral sidebands calculated by Eq. (16).

starts from noise, $M_{\mu \mathrm{BI}}$ should grow larger with increased integrated bandwidth. This behavior is shown in blue in Fig. 8. The slope of $M_{\mu \mathrm{BI}}$ with bandwidth is consistent with the value estimated from the bunch length. Thus, combining $M_{1}$ obtained from the first set of simulations with $M_{\mu \mathrm{BI}}$ obtained from the second set, the effective number of modes in the sidebands can be calculated directly with Eq. (16). This is shown in dot-dashed purple line in Fig. 8. These derived values are compared with the effective number of modes in the sidebands obtained from the simulations with both filtered seed from upstream and $\mu \mathrm{BI}$ in the beam, which is shown in Fig. 8 with the yellow dashed line. The good agreement between the simulations and theoretical model corroborates the posited relationship between the seed and the $\mu \mathrm{BI}$-induced sidebands statistics in Eq. (16).

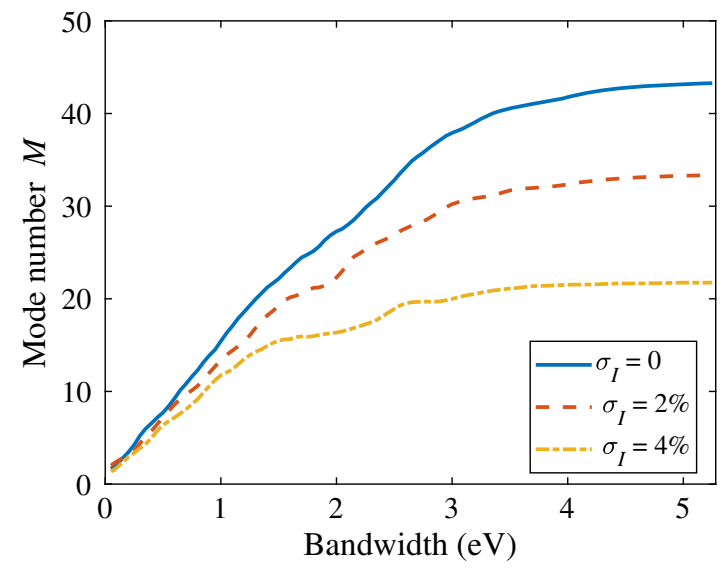

FIG. 9. The effective number of modes $M$ of the SASE output without $\mu \mathrm{BI}$ as a function of the integration bandwidth for different peak current fluctuations. 
Lastly, we performed numerical simulations to verify the effects of electron beam current fluctuations. For the pure SASE simulation without $\mu \mathrm{BI}$, we add controllable random variation to the peak current for each shot and analyze the output to get the number of modes, which is shown in Fig. 9. According to the simulation results, a $4 \%$ peak current fluctuation reduces the effective number of modes at full SASE bandwidth from 43 to 22. This is consistent with the theoretical prediction of Eq. (22). Using the parameters of the simulations in the next section, $I_{0}=1.3 \mathrm{kA}, \hat{z}=7$, we see that a $4 \%$ fluctuation in the peak current will reduce the effective number of modes from 43 to 24 .

\section{ANALYSIS OF A SELF-SEEDING EXPERIMENT AT LCLS}

For comparison with the analytic description and simulation results, we performed a statistical analysis on soft x-ray self-seeding (SXRSS) spectral data at LCLS $[5,8]$. Table III summarizes the typical electron beam and FEL operating parameters. The electron beam longitudinal phase space was optimized for producing narrow bandwidth SASE radiation from the first 6 or 7 undulator sections to maximize the monochromator throughput. Typical longitudinal phase spaces measurements made by the downstream X-band deflector [24] for lasing off and lasing on are shown in Fig. 10 together with current and slice energy spread profiles. The experiment controlled the level of the $\mu \mathrm{BI}$ in the electron beam by tuning the $\mathrm{LH}$ pulse energy $[22,25]$. Larger LH pulse energy results in weaker $\mu \mathrm{BI}$ in the electron beam. In the experiment, there are three LH pulse energies, $12 \mu \mathrm{J}$ (weak heating), $20 \mu \mathrm{J}$ (optimal heating) and $30 \mu \mathrm{J}$ (over heating).

The overall SXRSS experimental configuration, which occupies 9th undulator girder location (U9), can be found in Ref. [5]. The operating photon energy was $1 \mathrm{keV}$. The $200 \mathrm{meV}$ (FWHM) bandwidth of the monochromator is significantly smaller than the 1-2 eV FWHM SASE bandwidth at the end of U8. It is important to note that the associated Fourier limited pulse length of the seed is roughly 9 fs (FWHM), which is shorter than the length of

TABLE III. Experimental parameters of the soft X-ray selfseeding at the LCLS.

\begin{tabular}{lcc}
\hline \hline Parameter & Value & Unit \\
\hline Electron beam energy $E_{e}$ & 4.74 & $\mathrm{GeV}$ \\
Electron beam current $I$ & 1.3 & $\mathrm{kA}$ \\
Bunch charge $Q$ & 140 & $\mathrm{pC}$ \\
Slice energy spread $\sigma_{E}$ & 1.5 & $\mathrm{MeV}$ \\
Normalized emittance $\varepsilon_{N}$ & 0.6 & $\mu \mathrm{m}$ \\
Average focusing $\beta$ & 11 & $\mathrm{~m}$ \\
Seeding photon energy $h \nu$ & 1.0 & $\mathrm{keV}$ \\
Experimental gain length $L_{G}$ & 1.9 & $\mathrm{~m}$ \\
\hline \hline
\end{tabular}
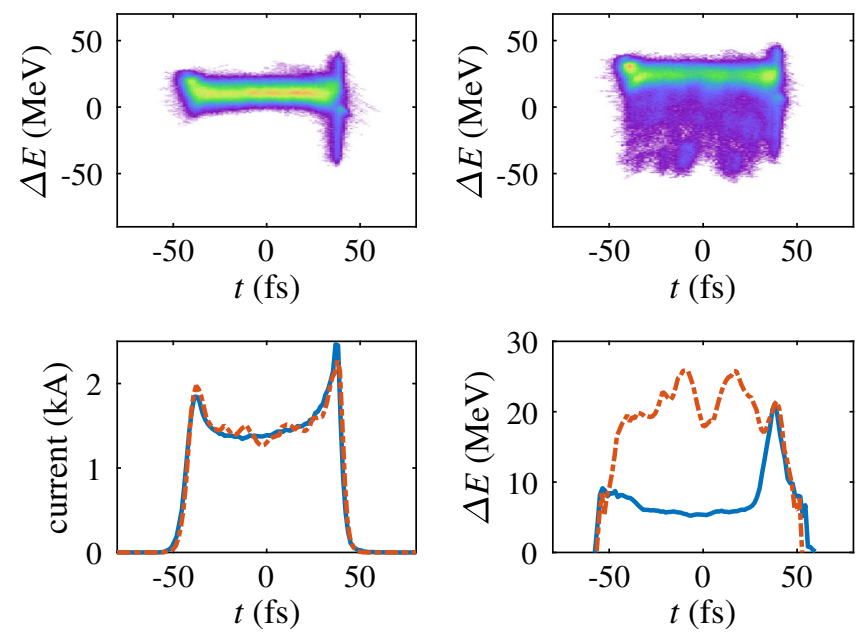

FIG. 10. Typical measured longitudinal phase space of the electron beam when lasing off (top left) and laser on (top right). Bottom-left: beam current profile and Bottom-right: slice energy spread profile of the two phase spaces for lasing off (solid line) and lasing on (dotted line).

the electron beam. Therefore, the system nominally seeds with multiple temporal and frequency spikes, i.e., consisting of several modes $\left(M_{1}>1\right)$.

The FEL output was measured at the effective undulator location U15 in November 2017 [8], which is approximately at the scaled position $\hat{z}=6-7$ in the second stage. The spectra of the self-seeding and SASE output were measured at three LH pulse energy settings: $12 \mu \mathrm{J}, 20 \mu \mathrm{J}$ and $30 \mu \mathrm{J}$. Figure 11 shows the average spectral intensity as a function of electron beam energy and photon energy. The FWHM resolution of the spectrometer is $\sim 270 \mathrm{meV}$ [8].
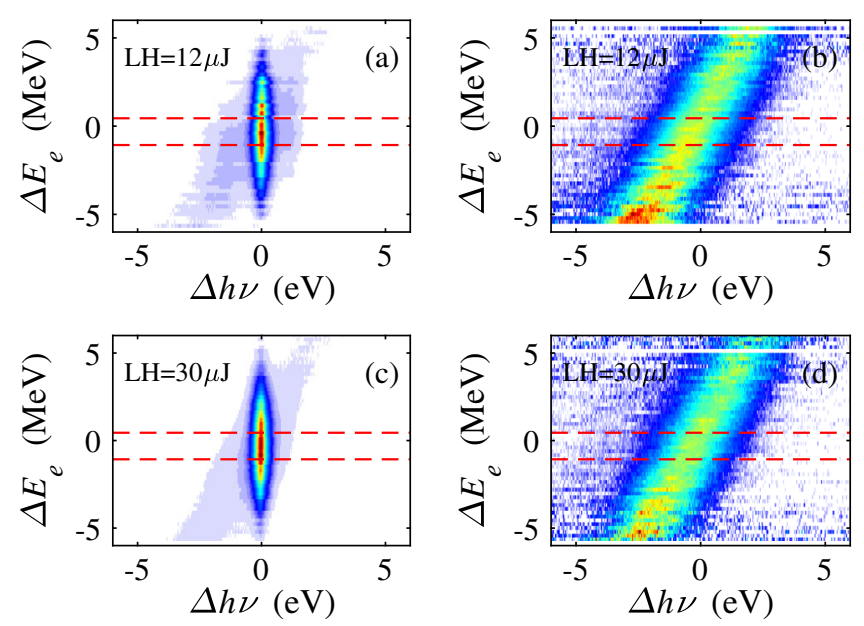

FIG. 11. Average spectral intensity as a function of electron beam energy and photon energy for different operation modes and laser heater $(\mathrm{LH})$ energies at U15. (a): self-seeding and LH $12 \mu \mathrm{J}$. (b): SASE and LH $12 \mu \mathrm{J}$. (c): self-seeding and LH $30 \mu \mathrm{J}$. (d): SASE and LH $30 \mu \mathrm{J}$. The red dashed lines denote the beam energy range used for the mode number analysis below. 


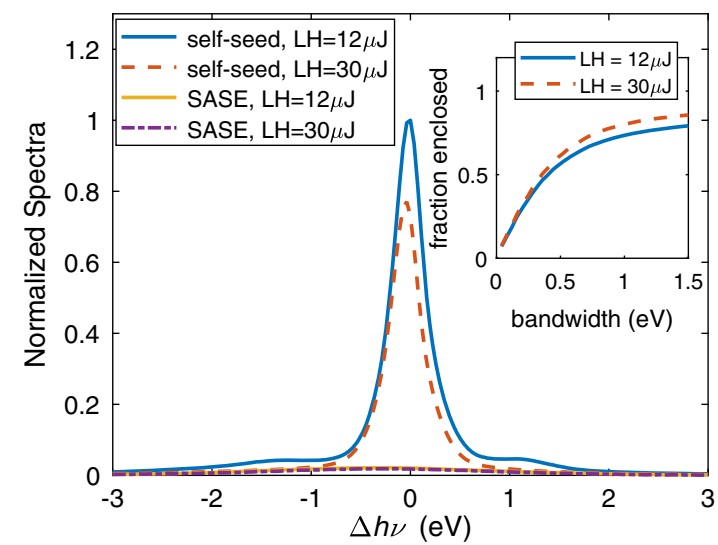

FIG. 12. Average spectra of the self-seeding and SASE output at U15 under different LH pulse energies. The inserted figure shows the fractional spectral energy as a function of integrated bandwidth.

The inherent electron beam energy jitter of the LCLS allows one to examine the correlation between the spectra and beam energy. It can be seen that the central wavelength of the self-seeding is very stable as the beam energy changes while the SASE spectra have a clear correlation with beam energy. As the LH pulse energy increases from 12 to $30 \mu \mathrm{J}$, the spectral pedestal is reduced significantly, especially on the low photon energy side of the seed.

For the statistical analysis we limit the electron beam energy range to near resonance as shown in Fig. 11 by interval enclosed within the red dashed lines. The average spectra of the self-seeding and SASE are shown in Fig. 12. The pedestal for the $12 \mu \mathrm{J} \mathrm{LH}$ energy is most apparent for photon energies offset about $-1.4 \mathrm{eV}$ from the primary seed spike. This figure also illustrates the effectiveness of the LH to suppress some of these features with the pedestal approaching the SASE limit for the $30 \mu \mathrm{J}$ energies. The inserted figure in Fig. 12 shows the spectral energy fraction as a function of bandwidth enclosed, centered at the seed photon energy. The inset also shows that the pedestal fraction decreases when increasing the LH pulse energy from $12 \mu \mathrm{J}$ to $30 \mu \mathrm{J}$.

The statistical analysis was performed on the measured spectra employing the same method used in the simulations. For the average self-seeded and SASE output spectra at $30 \mu \mathrm{J}$ LH pulse energy, the rms bandwidth from Gaussian fitting are $\sigma_{\text {seed }}=0.19 \mathrm{eV}$ and $\sigma_{\mathrm{SASE}}=$ $1.25 \mathrm{eV}$. Figure 13 displays histograms of the normalized integrated spectral intensity of the seed and SASE spectra over the full seed bandwidth $\left( \pm 3 \sigma_{\text {seed }}\right)$ and SASE bandwidth $\left( \pm 3 \sigma_{\mathrm{SASE}}\right)$, respectively. Empirically fit probability density functions are also shown. The effective mode number calculated from spectral fluctuation of the selfseeding and SASE output are 4.8 and 25.2 which are very close to the values (4.1 and 23.8) obtained from the $\Gamma$-distribution fitting. These values are both smaller than the ones given by the theory and simulations, unless
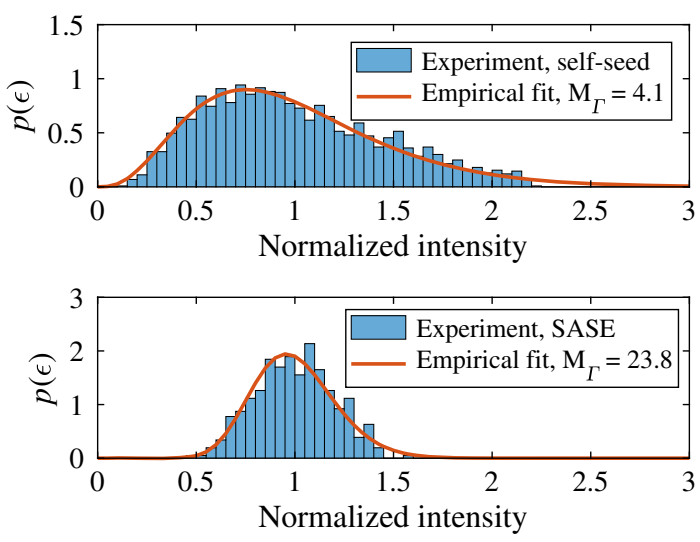

FIG. 13. Histograms of the normalized integrated spectral intensity (blue bars) and empirically derived probability density function (red curves) using Eq. (3) for the experimental measured self-seeding (top) and SASE (bottom) output at U15 with LH $30 \mu \mathrm{J}$.

fluctuations of the beam peak current are included. During the experiments, the electron beam current was monitored by the coherent radiation strength at the magnetic chicane, which shows $4 \%-5 \%$ peak current fluctuation in the shots used for analysis. The resulting reduction of the effective number of modes is consistent with the theory prediction and the simulation results as shown in Fig. 9.

The effective number of modes versus the integration bandwidth was calculated from the fluctuations in the integrated spectral energy under different LH energy settings, as shown in Fig. 14. With self-seeding one clearly sees that the slope of the curve changes near the bandwidth $\sim 0.4 \mathrm{eV}$, as predicted by theory and verified in the simulations. The effective number of modes in the SASE output increases almost linearly over the whole bandwidth range. Note that the effective number of modes of the SASE at very small bandwidth is about twice that of
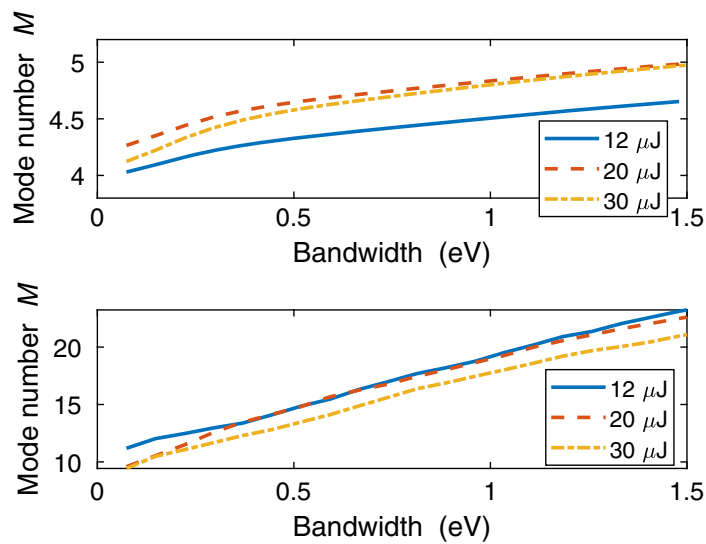

FIG. 14. The effective number of modes $\mathrm{M}$ as a function of the integration bandwidth for self-seeding (top) and SASE (bottom) under different LH energies at U15. The integration bandwidth is centered on the amplified seed wavelength. 
self-seeding, which probably comes from difference in the transverse coherence properties between the SASE and self-seeding, since measured SASE is produced only in the second stage and thus experiences about half the distance of exponential gain compared to the seed. The mode number calculated from the experimental spectra includes both longitudinal and transverse modes, while the 1D theoretical analysis and simulation results only focus on the longitudinal modes. According to previous simulation and experimental studies at the LCLS [26,27], the transverse coherence of the SASE is about half that of self-seeding at this $\hat{z}=6-7$ position in the gain curve (saturation occurs around $\hat{z}=9$ ), taking lethargy into account.

From the plots in Fig. 14, we see that experimentally the LH has little effect on either the seed or SASE statistics. Compared with the simulations, the lack of LH impact on the SASE in particular is likely because the level of $\mu \mathrm{BI}$ in the experiments was much smaller, so the effect may not be resolved.

Regarding the spectral pedestal, however, there are significant changes in the effective number of modes as the LH power is increased. These are shown in Fig. 15, where the integration bandwidth is offset $-1.4 \mathrm{eV}$. When the LH pulse energy is low (i.e., $12 \mu \mathrm{J}$ ), the effective mode number of the pedestal is very close to that of the seed, i.e., $M \approx 3-4$. Increasing the pulse energy to $30 \mu \mathrm{J}$, we can see the effective number of modes approach that of SASE. The behavior of the statistical properties of the measured spectral pedestal then agrees well with the theory and simulations in the previous sections, pointing to the role that $\mu \mathrm{BI}$ plays as a source of the spectral pedestal in the self-seeding output.

Finally, as predicted by the theoretical analysis, we can connect the effective number of modes of the spectral pedestal with $\eta$, the ratio of $\mu \mathrm{BI}$-induced sidebands to SASE background. For the measured self-seeding spectra, if we limit the peak intensity of the seed signal to a

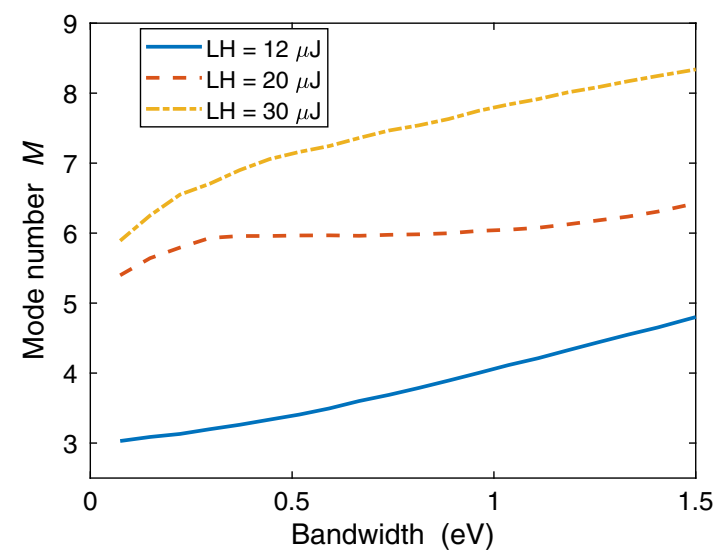

FIG. 15. The effective number of modes $M$ as a function of the integration bandwidth for self-seeding spectral pedestals under different LH energies at U15. The integration bandwidth is offset $-1.4 \mathrm{eV}$ from the seed center as measured in the spectra of Fig. 12.

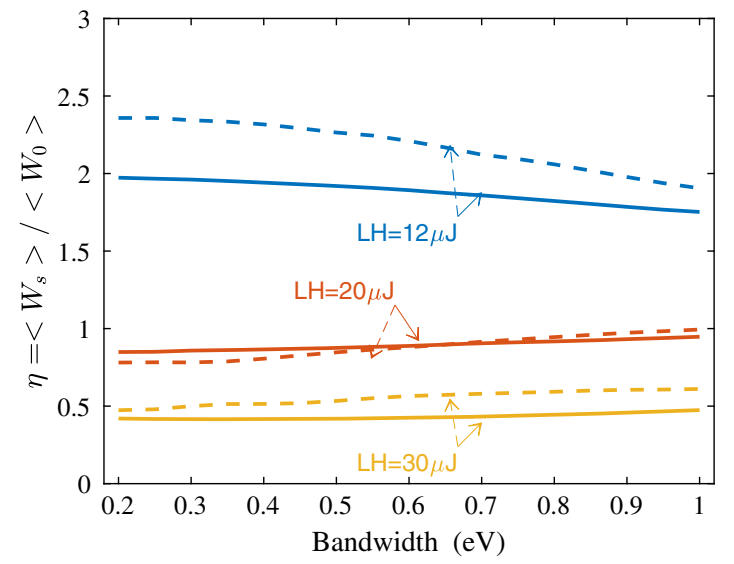

FIG. 16. Comparisons of the ratios of the $\mu$ BI-induced sidebands and SASE background versus integration bandwidth under different LH settings at U15. The ratios are calculated by the deduction of measured spectra (solid line) and the number of modes in statistical analysis (dashed line). The integration bandwidth is again offset $-1.4 \mathrm{eV}$ from the seed center.

relatively narrow range (i.e., stabilizing the seed), the spectral pedestal can be viewed approximately as the sum of the $\mu \mathrm{BI}$-induced sidebands with a SASE background and a constant seed. Based on this approximation, we can derive the values of $M_{\mu \mathrm{BI}}$ at different integration bandwidths. In this case, using Eqs. (16) and (17), we can solve for $\eta$. On the other hand, $\eta$ can also be obtained by directly comparing the average spectra of self-seeding and SASE. Figure 16 shows the comparisons of $\eta$ calculated by these two methods under different LH settings. There is good agreement between the two approaches, especially at higher LH energies. Again, this indicates that the theoretical treatment effectively captures the various statistical contributions to the spectral pedestal in self-seeding.

\section{CONCLUSION}

We have studied the statistical properties of a self-seeded XFEL, mainly focusing on the number of modes of the seed and spectral pedestal, and how they are related. It is believed that the pedestals in the measured spectra are often dominated by uBI-induced sidebands. A theoretical model was developed based on spectral effects produced both by SASE and $\mu \mathrm{BI}$ sidebands in seeded FELs, and used to explain the behavior of the statistical properties of the spectral pedestal under different $\mu \mathrm{BI}$ and seeding conditions. The effective number of modes was defined by the fluctuation of the integrated energy within a variable bandwidth, which can straightforwardly be extended to the sum or product of several sources. The theoretical model matched well with numerical simulations, including the number of modes in the main seed and the spectral pedestal as functions of the integration bandwidth. The effective number of modes of the seed first increases rapidly within the seed bandwidth and then more slowly 
with the inclusion of more SASE radiation. When the $\mu \mathrm{BI}-$ induced modulations in the electron beam are small, for example, when suppressed by the LH, the effective number of modes of the pedestal is close to that of the SASE background. On the other hand, when the pedestal is dominated by the $\mu \mathrm{BI}$-induced sidebands, the effective number of modes in the pedestal is more strongly related to the effective number of seed modes. The fluctuation of the pedestal reflects the level of $\mu \mathrm{BI}$ in the beam, but does not cause significant fluctuations in the seed. The agreement between the theoretical model and the experimental results further verifies the $\mu \mathrm{BI}$-induced sideband as a main source of the spectral pedestal in self-seeded XFELs.

\section{ACKNOWLEDGMENTS}

We thank our colleagues Y. Ding, D. Ratner and J. Krzywinski for useful discussions. This work was supported by U.S. Department of Energy Contract No. DE-AC02-76SF00515 and Award No. 2017-SLAC100382.

\section{APPENDIX}

In this Appendix, we present the derivation of the effective number of sideband modes. As the sideband is driven by the seed and the $\mu \mathrm{BI}$-induced modulations, we will have

$$
W_{s}=a W_{1} A^{2},
$$

where $a$ is the coupling coefficient. Here we assume the sideband source (e.g., $\mu \mathrm{BI}$ modulations) and the seed strength are uncorrelated. So the variance of $M_{s}$ can be written as

$$
\begin{aligned}
\frac{\operatorname{Var}\left(W_{s}\right)}{a^{2}} & =E\left(W_{1}^{2} A^{4}\right)-\left[E\left(W_{1} A^{2}\right)\right]^{2} \\
& =E\left(W_{1}^{2}\right) E\left(A^{4}\right)-\left[E\left(W_{1}\right)\right]^{2}\left[E\left(A^{2}\right)\right]^{2} \\
& =\left\{\operatorname{Var}\left(W_{1}\right)+\left[E\left(W_{1}\right)\right]^{2}\right\}\left\{\operatorname{Var}\left(A^{2}\right)+\left[E\left(A^{2}\right)\right]^{2}\right\}-\left[E\left(W_{1}\right)\right]^{2}\left[E\left(A^{2}\right)\right]^{2} \\
& =\operatorname{Var}\left(W_{1}\right) \operatorname{Var}\left(A^{2}\right)+\operatorname{Var}\left(W_{1}\right)\left[E\left(A^{2}\right)\right]^{2}+\operatorname{Var}\left(A^{2}\right)\left[E\left(W_{1}\right)\right]^{2}
\end{aligned}
$$

Here $E(W)$ means the expectation of the variable $W$. The effective numbers of modes are defined as

$$
\begin{gathered}
M_{s}=\frac{\left[E\left(W_{s}\right)\right]^{2}}{\operatorname{Var}\left(W_{s}\right)}, \\
M_{1}=\frac{\left[E\left(W_{1}\right)\right]^{2}}{\operatorname{Var}\left(W_{1}\right)}, \\
M_{\mu \mathrm{BI}}=\frac{\left[E\left(A^{2}\right)\right]^{2}}{\operatorname{Var}\left(A^{2}\right)} .
\end{gathered}
$$

Replacing all the variances by the product of the expectation and the number of modes and using the fact that $E\left(a W_{1} W_{\mu \mathrm{BI}}\right)=a E\left(W_{1}\right) E\left(W_{\mu \mathrm{BI}}\right)$, we can obtain

$$
\frac{1}{M_{s}}=\frac{1}{M_{1}}+\frac{1}{M_{\mu \mathrm{BI}}}+\frac{1}{M_{1} M_{\mu \mathrm{BI}}} .
$$

[1] J. Feldhaus, E. L. Saldin, J. R. Schneider, E. A. Schneidmiller, and M. V. Yurkov, Possible application of $\mathrm{X}$-ray optical elements for reducing the spectral bandwidth of an X-ray sase fel, Nucl. Instrum. Methods Phys. Res., Sect. A 393, 162 (1997).

[2] G. Geloni, V. Kocharyan, and E. Saldin, A novel selfseeding scheme for hard x-ray fels, J. Mod. Opt. 58, 1391, 2011.

[3] J. Amann, W. Berg, V. Blank, F.-J. Decker, Y. Ding, P. Emma, Y. Feng, J. Frisch, D. Fritz, J. Hastings et al., Demonstration of self-seeding in a hard-X-ray free-electron laser, Nat. Photonics 6, 693 (2012).

[4] A. A. Lutman, F.-J. Decker, J. Arthur, M. Chollet, Y. Feng, J. Hastings, Z. Huang, H. Lemke, H.-D. Nuhn, A. Marinelli et al., Demonstration of Single-Crystal Self-Seeded Two-Color X-Ray Free-Electron Lasers, Phys. Rev. Lett. 113, 254801 (2014).

[5] D. Ratner, R. Abela, J. Amann, C. Behrens, D. Bohler, G. Bouchard, C. Bostedt, M. Boyes, K. Chow, D. Cocco et al., Experimental Demonstration of a Soft X-Ray Self-Seeded Free-Electron Laser, Phys. Rev. Lett. 114, 054801 (2015).

[6] C. Emma, A. Lutman, M. W. Guetg, J. Krzywinski, A. Marinelli, J. Wu, and C. Pellegrini, Experimental demonstration of fresh bunch self-seeding in an X-ray free electron laser, Appl. Phys. Lett. 110, 154101 (2017).

[7] I. Inoue, T. Osaka, T. Hara, T. Tanaka, T. Inagaki, T. Fukui, S. Goto, Y. Inubushi, H. Kimura, R. Kinjo et al., Generation of narrow-band $\mathrm{X}$-ray free-electron laser via reflection self-seeding, Nat. Photonics 13, 319 (2019).

[8] G. Marcus, W. M. Fawley, D. Bohler, Y. Ding, Y. Feng, E. Hemsing, Z. Huang, J. Krzywinski, A. Lutman, and D. Ratner, Experimental observations of seed growth and accompanying pedestal contamination in a self-seeded, soft 
X-ray free-electron laser, Phys. Rev. Accel. Beams 22, 080702 (2019).

[9] E. L. Saldin, E. A. Schneidmiller, and M. V. Yurkov, Klystron instability of a relativistic electron beam in a bunch compressor, Nucl. Instrum. Methods Phys. Res., Sect. A 490, 1 (2002).

[10] Z. Huang, M. Borland, P. Emma, J. Wu, C. Limborg, G. Stupakov, and J. Welch, Suppression of microbunching instability in the linac coherent light source, Phys. Rev. Accel. Beams 7, 074401 (2004).

[11] Z. Zhang, R. Lindberg, W. M. Fawley, Z. Huang, J. Krzywinski, A. Lutman, G. Marcus, and A. Marinelli, Microbunching-instability-induced sidebands in a seeded free-electron laser, Phys. Rev. Accel. Beams 19, 050701 (2016).

[12] K. Zhang, L. Zeng, Z. Qi, C. Feng, and D. Wang, Eliminating the microbunching-instability-induced sideband in a soft X-ray self-seeding free-electron laser, Nucl. Instrum. Methods Phys. Res., Sect. A 882, 22 (2018).

[13] E. L. Saldin, E. A. Schneidmiller, and M. V. Yurkov, Statistical properties of radiation from vuv and x-ray free electron laser, Opt. Commun. 148, 383 (1998).

[14] E. L. Saldin, E. A. Schneidmiller, and M. V. Yurkov, Coherence properties of the radiation from $\mathrm{X}$-ray free electron laser, Opt. Commun. 281, 1179 (2008).

[15] E. L. Saldin, E. V. Schneidmiller, and M. V. Yurkov, The Physics of Free Electron Lasers (Springer Science \& Business Media, New York, 2013).

[16] S. Krinsky and Y. Li, Statistical analysis of the chaotic optical field from a self-amplified spontaneous-emission free-electron laser, Phys. Rev. E 73, 066501 (2006).

[17] A. Lutman, Z. Huang, J. Krzywinski, J. Wu, D. Zhu, and Y. Feng, Statistical characterization of an X-ray fel in the spectral domain, in Advances in X-ray Free-Electron Lasers Instrumentation IV (International Society for Optics and Photonics, SPIE, 2017), Vol. 10237, p. 102370H.

[18] J. W. Goodman, Statistical Optics, 2nd ed. (John Wiley \& Sons, New York, 2015).
[19] E. Hemsing, A. Halavanau, and Z. Zhang, Statistical theory of a self-seeded free electron laser with noise pedestal growth, Phys. Rev. Accel. Beams 23, 010701 (2020).

[20] R. Bonifacio, C. Pellegrini, and L. M. Narducci, Collective instabilities and high-gain regime in a free electron laser, Opt. Commun. 50, 373 (1984).

[21] M. V. Yurkov, Statistical properties of sase fel radiation: experimental results from the vuv fel at the tesla test facility at desy, Nucl. Instrum. Methods Phys. Res., Sect. A 483, 51 (2002).

[22] D. Ratner, C. Behrens, Y. Ding, Z. Huang, A. Marinelli, T. Maxwell, and F. Zhou, Time-resolved imaging of the microbunching instability and energy spread at the linac coherent light source, Phys. Rev. Accel. Beams 18, 030704 (2015).

[23] A. W. Chao, Physics of Collective Beam Instabilities in High Energy Accelerators (Wiley, New York, 1993).

[24] C. Behrens, F.-J. Decker, Y. Ding, V. A. Dolgashev, J. Frisch, Z. Huang, P. Krejcik, H. Loos, A. Lutman, T. J. Maxwell et al., Few-femtosecond time-resolved measurements of x-ray free-electron lasers, Nat. Commun. 5, 3762 (2014).

[25] Z. Huang, A. Brachmann, F.-J. Decker, Y. Ding, D. Dowell, P. Emma, J. Frisch, S. Gilevich, G. Hays, P. Hering et al., Measurements of the linac coherent light source laser heater and its impact on the X-ray free-electron laser performance, Phys. Rev. Accel. Beams 13, 020703 (2010).

[26] Y. Ding, Z. Huang, S. A. Ocko et al., Transverse-coherence properties of the fel at the LCLS, in Proceedings of the 32nd Free Electron Laser Conference, Malmöö, Sweden (Max-lab, Sweden, 2010).

[27] I. A. Vartanyants, A. Singer, A. P. Mancuso, O. M. Yefanov, A. Sakdinawat, Y. Liu, E. Bang, G. J Williams, G. Cadenazzi, B. Abbey et al., Coherence Properties of Individual Femtosecond Pulses of an X-Ray Free-Electron Laser, Phys. Rev. Lett. 107, 144801 (2011). 